\title{
Beam halo definitions based upon moments of the particle distribution
}

\author{
C. K. Allen and T. P. Wangler \\ Los Alamos National Laboratory, Los Alamos, New Mexico 87545
}

(Received 23 July 2002; published 20 December 2002)

\begin{abstract}
Two different parameters for the quantitative description of beam halo are discussed. Both are based on moments of the particle distribution and represent a convenient and model-independent method for quantifying the magnitude of beam halo observed in either spatial or phase-space projections. One parameter is a measure of spatial profile of the beam and has been defined by Wangler and Crandall previously. The current authors defined a new parameter using kinematic invariants to quantify halo formation in 2D phase space. Here we expand the development and present detailed numerical results. Although the spatial-profile parameter and the phase-space halo parameter both reduce to the same value when the distribution has the elliptical symmetry, in general these parameters are not equal. Halo in the 1D spatial profiles is relatively easily measured, but is variable as the beam distribution evolves and can hide as it rotates in phase space. The 2D phase-space halo is more difficult to measure, but it varies more smoothly as the halo evolves. It provides a more reliable characterization of the halo as an intrinsic property of the beam.
\end{abstract}

DOI: 10.1103/PhysRevSTAB.5.124202

PACS numbers: 29.17.+w, 29.27.Bd, 41.75.-i

\section{INTRODUCTION}

The existence of beam halo is an important characteristic of high-intensity beams [1-5]. Although by visual inspection of measured or simulated particle distributions, we can intuitively identify halo, it is important to obtain a more quantitative measure of this phenomenon. We have been investigating parameters based on moments of the particle distribution that provide a quantitative description of halo. We are looking for a parameter that describes halo in a similar vein with the most important figure of merit of beam quality, the rms emittance. The parameter should reflect the identifying characteristics of halo and be useful for both theory and experiment. Our current conclusions and findings are presented herein.

We find that halo, like rms emittance, is an intrinsic property of the beam by introducing the halo parameter $H$ that identifies the amount of halo in a particular beam distribution. The halo parameter contains additional information as to the beam state, since we find that it is possible to have emittance growth without halo growth (however, halo growth always implies emittance growth). As with emittance, the halo parameter is invariant under linear forces. Thus, halo growth is necessarily the result of nonlinearities.

Wangler and Crandall proposed a quantity for characterization of halo in a 1D spatial projection, called the beam profile parameter, constructed from the second and fourth spatial moments of the beam [6]. It is important to have a definition of halo in the 1D spatial projection for which experimental measurements are relatively easy to obtain. Through simulation studies, they found that the parameter was indeed a good indicator of the visually observable halo. However, because of the beam distribution's phase-space rotations, the observed halo in 1D oscillates, so that halo at different locations along the beam line is observable in differing degrees. For example, at some locations the halo may project strongly along the spatial coordinate and only weakly along the momentum coordinate, while at others the reverse is true, and the halo can be hidden in the spatial projection. In most circumstances, the beam halo from simulation appears as an irreversible effect, when observed in the 2D phase-space distributions. Therefore, it is also important to search for another definition of halo in the 2D phase-space distributions. In this case the halo is not expected to change as the beam distribution rotates in phase space, and the halo parameter in $2 \mathrm{D}$ phase space represents a measure of the halo as an intrinsic property of the beam.

Our approach here is to extend the 1D work to obtain a halo parameter based on moments of the particle distribution suitable for description of the beam halo in 2D phase space. In so doing, one is led naturally to the moment invariants presented by Lysenko [7,8] and Dragt [9]. Specifically, these are polynomial functions of the distribution moments, which are invariant whenever all the forces on the beam are linear (including self-forces). These quantities are known as kinematic invariants and are the consequence of the linear forces and symplectic structure imposed by Hamilton's equations. Any quantity built from the kinematic invariants would vary only in situations where nonlinear forces were present, including nonlinear self-forces. For example, the rms emittance is known to have these properties and is indeed a kinematic invariant. We first introduced this idea of halo quantification based on kinematic invariants at the PAC2001 conference [10]; here we expand the development and present detailed numerical simulation results. 


\section{HALO PARAMETERS}

There is no clearly defined separation between the halo and the main core of the beam. Consequently, there has been some difficulty identifying a suitable quantitative measure of the halo content of a beam in a modelindependent way. We consider two parameters for quantifying the halo, based on moments of the particle distribution. Wangler and Crandall originally defined the spatial profile parameter $h$ for the 1D spatial projections [6]. We introduce the phase-space halo parameter $H$ for the $2 \mathrm{D}$ phase-space distributions. The halo parameter generalizes the spatial-profile parameter using kinematic invariants of the particle distribution in phase space. We shall also consider separately the different cases of continuous beams and bunched beams. Different definitions of $h$ and $H$ are provided for each case.

\section{A. The spatial-profile parameter for continuous beams}

A general characteristic of beam halo is the increased population of the outer part of the beam. The spatialprofile parameter describes this feature in coordinate space. Let the coordinates of the $i$ th phase plane be denoted $\left(q_{i}, p_{i}\right)$, where $q_{i}$ and $p_{i}$ are the spatial and momentum coordinates, respectively. The spatial-profile parameter, denoted $h_{i}$, is then defined for continuous beams as [6]

$$
h_{i} \equiv \frac{\left\langle q_{i}^{4}\right\rangle}{\left\langle q_{i}^{2}\right\rangle^{2}}-2,
$$

where $\langle\cdot\rangle$ is the moment operator or average over the particle distribution. Note that $h_{i}$ involves only spatial moments of the distribution. The constant in the above definition is chosen to normalize the parameter so that it has the value 0 for a Kapchinskij-Vladmirskij (KV) distribution for which there is no halo. For a Gaussian distribution $h_{i}=1$. Multiparticle simulations show that significant halo presence in a $1 \mathrm{D}$ projection corresponds to $h_{i}>1$ (see Ref. [6]).

Notice that the definition of $h$ is essentially the kurtosis of the beam where we have used the value 2 instead of 3 in the standard definition. Kurtosis is typically used to compare the "peakedness" of a distribution to that of a Gaussian. Flatter distributions have negative kurtosis while sharper ones have positive kurtosis. By using the value 2 we are essentially normalizing to a uniform distribution rather than a Gaussian; this seems more appropriate for beam dynamics.

\section{B. The phase-space halo parameter for continuous beams}

To describe presence of halo in 2D phase space we introduce the beam halo parameter $H$. If the motion is uncoupled between phase planes, the following quantities are kinematic invariants of motion [9]:

$$
\begin{aligned}
I_{2}^{i} & \equiv\left\langle q_{i}^{2}\right\rangle\left\langle p_{i}^{2}\right\rangle-\left\langle q_{i} p_{i}\right\rangle^{2}, \\
I_{4}^{i} & \equiv\left\langle q_{i}^{4}\right\rangle\left\langle p_{i}^{4}\right\rangle+3\left\langle q_{i}^{2} p_{i}^{2}\right\rangle^{2}-4\left\langle q_{i} p_{i}^{3}\right\rangle\left\langle q_{i}^{3} p_{i}\right\rangle .
\end{aligned}
$$

Then we define the halo intensity parameter, $H_{i}$, in the $i$ th phase plane, as

$$
\begin{aligned}
H_{i} & \equiv \frac{\sqrt{3 I_{4}^{i}}}{2 I_{2}^{i}}-2 \\
& =\frac{\sqrt{3\left\langle q_{i}^{4}\right\rangle\left\langle p_{i}^{4}\right\rangle+9\left\langle q_{i}^{2} p_{i}^{2}\right\rangle^{2}-12\left\langle q_{i} p_{i}^{3}\right\rangle\left\langle q_{i}^{3} p_{i}\right\rangle}}{2\left\langle q_{i}^{2}\right\rangle\left\langle p_{i}^{2}\right\rangle-2\left\langle q_{i} p_{i}\right\rangle^{2}}-2 .
\end{aligned}
$$

The constants are chosen for normalization consistent with that used for the spatial-profile parameter. Thus, in situations of elliptical symmetry in phase space, $H_{i}$ will have a value 0 for the $K V$ distribution and a value 1 for the Gaussian distribution. Multiparticle simulations show that significant halo in the 2D phase-space projection corresponds to $H_{i}>1$.

\section{Comparison of the parameters for beams with elliptical symmetry}

For idealized beam distributions with elliptically symmetric isodensity contours in $2 \mathrm{D}$ phase space the projected density function $\rho(q, p)$ has the form

$$
\rho(q, p)=f\left(\gamma q^{2}+2 \alpha q p+\beta p^{2}\right),
$$

where $\alpha, \beta$, and $\gamma$ are the Courant-Snyder parameters and $f(\cdot)$ is a real, positive function. The moments of this distribution may be computed analytically in terms of the moments of $f$ (see the Appendix). For example, we have

$$
\begin{aligned}
& \left\langle q^{2}\right\rangle=\frac{1}{2} \beta \frac{F_{1}}{F_{0}}, \quad\left\langle p^{2}\right\rangle=\frac{1}{2} \gamma \frac{F_{1}}{F_{0}}, \quad\langle q p\rangle=-\frac{1}{2} \alpha \frac{F_{1}}{F_{0}}, \quad\left\langle q p^{3}\right\rangle=-\frac{3}{8} \alpha \gamma \frac{F_{2}}{F_{0}}, \quad\left\langle q^{4}\right\rangle=\frac{3}{8} \beta^{2} \frac{F_{2}}{F_{0}}, \\
& \left\langle p^{4}\right\rangle=\frac{3}{8} \gamma^{2} \frac{F_{2}}{F_{0}}, \quad\left\langle q^{2} p^{2}\right\rangle=\left(\frac{3 \alpha^{2}}{8}+\frac{1}{8}\right) \frac{F_{2}}{F_{0}}, \quad\left\langle q^{3} p\right\rangle=-\frac{3}{8} \alpha \beta \frac{F_{2}}{F_{0}},
\end{aligned}
$$

where

$$
\begin{aligned}
& F_{0} \equiv \int_{0}^{\infty} f(s) d s, \quad F_{1} \equiv \int_{0}^{\infty} s f(s) d s, \\
& F_{2} \equiv \int_{0}^{\infty} s^{2} f(s) d s
\end{aligned}
$$

or, more generally,

$$
F_{n} \equiv \int_{0}^{\infty} s^{n} f(s) d s
$$

Using these relations, we find the following relationship for the profile and halo parameters: 


$$
h=H=\frac{3}{2} \frac{\left(F_{2} / \mathrm{F}_{0}\right)}{\left(F_{1} / \mathrm{F}_{0}\right)^{2}}-2 .
$$

Thus, for elliptically symmetric distributions satisfying Eq. (4), the profile and halo parameters are analytically equivalent. The halo parameter can consequently be considered a generalization of the kurtosis of a particle beam. However, more general beam distributions from multiparticle simulations do not have elliptically symmetric isodensity contours, especially in the outer regions where the halo is concentrated, and Eq. (8) is not satisfied.

For an idealized beam with elliptical symmetry, Eq. (8) has another interesting interpretation. Let $\varepsilon$ be the emittance associated with any elliptical isodensity contour. Then we have the following relation:

$$
\varepsilon \equiv \gamma q^{2}+2 \alpha q p+\beta p^{2},
$$

so that $\pi \varepsilon$ is the area of the phase-space ellipse identified by the above equation. The first two moments of the contour emittance are given by

$$
\bar{\varepsilon} \equiv\langle\varepsilon\rangle=\frac{\int_{0}^{\infty} \varepsilon f(\varepsilon) d \varepsilon}{\int_{0}^{\infty} f(\varepsilon) d \varepsilon}, \quad \tilde{\varepsilon}^{2} \equiv\left\langle\varepsilon^{2}\right\rangle=\frac{\int_{0}^{\infty} \varepsilon^{2} f(\varepsilon) d \varepsilon}{\int_{0}^{\infty} f(\varepsilon) d \varepsilon} .
$$

Therefore, the profile and halo parameters appear as

$$
\begin{aligned}
H & =h=\frac{3}{2} \frac{\left\langle\varepsilon^{2}\right\rangle}{\langle\varepsilon\rangle^{2}}-2=\frac{3}{2} \frac{\tilde{\varepsilon}^{2}}{\bar{\varepsilon}^{2}}-2, \\
& =\frac{3}{2}\left(\frac{\sigma_{\varepsilon}^{2}}{\bar{\varepsilon}^{2}}-\frac{1}{3}\right),
\end{aligned}
$$

where $\bar{\varepsilon}$ and $\sigma_{\varepsilon}^{2}=\left\langle(\varepsilon-\bar{\varepsilon})^{2}\right\rangle=\tilde{\varepsilon}^{2}-\bar{\varepsilon}^{2}$ are the average value and variance of $\varepsilon$, respectively. Thus for elliptical symmetry, both parameters are linearly proportional to the ratio of the standard deviation of the contour emittance to the squared average value of the contour emittance.

\section{Six-dimensional phase space: bunched beams}

Wangler and Crandall provided a different normalization constant for bunched beams. The constant $15 / 7$ was chosen so that the profile parameter $h_{i}$ would be zero for a uniform density bunch in $x, y, z$ space. The resulting spatial-profile parameter definition is

$$
h_{i} \equiv \frac{\left\langle q_{i}^{4}\right\rangle}{\left\langle q_{i}^{2}\right\rangle^{2}}-\frac{15}{7} .
$$

Likewise, for bunched beams we define the phase-space halo parameter $H_{i}$ as

$$
H_{i} \equiv \frac{\sqrt{3}}{2} \frac{\sqrt{I_{4}^{i}}}{I_{2}^{i}}-\frac{15}{7} .
$$

Note that the bunched-beam constant $15 / 7 \approx 2.143$ is close to the continuous-beam value of 2 .
Next, we investigate the halo parameters for bunched beams with ellipsoidally symmetric isodensity contours. For ellipsoidal symmetry in six-dimensional phase space, the distribution $\rho$ has the form

$$
\rho\left(q_{1}, p_{1}, q_{2}, p_{2}, q_{3}, p_{3}\right)=q f\left(z^{T} Q z\right)
$$

where

$$
z \equiv\left(q_{1} p_{1} q_{2} p_{2} q_{3} p_{3}\right)^{T},
$$

$f$ is a positive real function, and $Q$ is some symmetric, positive definite, real $6 \times 6$ matrix (generalized CourantSnyder parameters). For distributions described by Eq. (14), it is again possible to compute analytically the values of the profile and halo parameters, $H_{i}$ and $h_{i}$. We first compute the invariants $I_{2}^{i}$ and $I_{4}^{i}$. They depend upon the determinant of $Q$, having the values

$$
I_{2}^{i}=\frac{1}{36} \frac{F_{3}^{2}}{F_{2}^{2}} \frac{1}{\operatorname{det} Q}, \quad I_{4}^{i}=\frac{1}{192} \frac{F_{4}^{2}}{F_{2}^{2}} \frac{1}{(\operatorname{det} Q)^{2}} .
$$

However, the halo and profile parameters depend only upon the distribution function $f$

$$
H_{i}=h_{i}=\frac{9}{4} \frac{F_{4} \mathrm{~F}_{2}}{F_{3}^{2}}-\frac{15}{7} .
$$

For the case of ellipsoidal symmetry in 6D space, we find that both parameters are again equal. Generally, beam distributions from multiparticle simulations do not have ellipsoidally symmetric isodensity contours, and Eq. (17) is not satisfied.

\section{NUMERICAL VALUES}

To illustrate typical behavior of the profile and halo parameters we present some numerical values for particular beam distributions. We include both analytic distributions commonly used in theoretical developments and distributions obtained from numerical simulation.

\section{A. Parameter values for common phase-space distributions}

We tabulate the values of the profile and halo parameters for several standard analytic distributions generally considered not to have significant halo. Table I lists the

TABLE I. Halo parameter values for some common analytic distributions.

\begin{tabular}{lcc}
\hline \hline Distribution & $\begin{array}{c}H, h \\
\text { Continuous }\end{array}$ & Bunched \\
\hline Uniform & 0 & 0 \\
Parabolic & $1 / 4$ & $4 / 21$ \\
Gaussian & 1 & $6 / 7$ \\
Hollow & $1 / 4$ & $75 / 112$ \\
\hline \hline
\end{tabular}


TABLE II. Profile parameter values for 1D distributions.

\begin{tabular}{|c|c|c|}
\hline Distribution & $f(x)$ & $h$ \\
\hline Triangular & $f(x)= \begin{cases}(1 / \xi)(1-x / \xi) & \text { for } x \in(0,+\xi] \\
(1 / \xi)(1+x / \xi) & \text { for } x \in[-\xi, 0]\end{cases}$ & $2 / 5$ \\
\hline Cusp & $f(x)=\frac{1}{2 \alpha} e^{-|x| / \alpha}$ & 4 \\
\hline Gaussian & $f(x)=\frac{1}{\sqrt{2 \pi} \sigma} e^{-\left[\left(x^{2}\right) /\left(2 \sigma^{2}\right)\right]}$ & $\begin{aligned} & 1 \\
\Gamma^{2}(1 / 4) & \end{aligned}$ \\
\hline "Hyper-Gaussian" & $f(x)=\frac{\sqrt{2}}{\Gamma(1 / 4) \kappa} e^{-\left[\left(x^{4}\right) /\left(4 \kappa^{4}\right)\right]}$ & $\frac{17}{4 \Gamma^{2}(3 / 4)}$ \\
\hline Parabolic & $f(x)= \begin{cases}\frac{3}{4 L}\left[1-(x / L)^{2}\right] & \text { for } x \in[-L,+L] \\
0 & \text { otherwise }\end{cases}$ & $1 / 7$ \\
\hline Uniform & $f(x)= \begin{cases}1 / L & \text { for } x \in[-L,+L] \\
0 & \text { otherwise }\end{cases}$ & $-1 / 5$ \\
\hline Hollow & $f(x)=\frac{1}{\sqrt{2 \pi} \sigma^{3}} x^{2} e^{-\left[\left(x^{2}\right) /\left(2 \sigma^{2}\right)\right]}$ & $-1 / 3$ \\
\hline
\end{tabular}

values of $h$ and $H$ both for the continuous case and for the bunched-beam case. In the continuous beam case, we assume the uniform beam has $2 \mathrm{D}$ projections that are uniform in any direction. For the bunched-beam case, the uniform beam is assumed to have uniform 3D projections in any direction. The functional form of each distribution is given in Table II. Notice that all values lie between 0 and 1, unlike beams from multiparticle simulations that show strong halo.

\section{B. Profile parameter values for 1D projections}

Table II shows the values of the profile parameter for one-dimensional distributions, that is, spatial density distributions. Distributions of this type are obtained from experimental measurements. In the values below, Eq. (1), the continuous-beam definition for $h$, was used. Note that it is possible to obtain negative values for $h$ in the onedimensional case.

\section{Numerical simulations}

To illustrate the behavior of the halo parameters $h$ and $H$ for distributions in multiparticle simulations, we consider simulations of the halo experiment at the Los Alamos Low-Energy Demonstration Accelerator. The Halo Experiment is designed to produce halo by mismatching the beam into a 52-quadrupole periodic FODO lattice. The beam is initially bunched; however, since there is no longitudinal focusing, it debunches as it propagates down the channel. Throughout the examples, we use the profile parameter $h_{i}$ defined by Eq. (1), and the halo parameter $H_{i}$ defined by Eq. (3).

We consider three cases: (i) the matched case, (ii) a mismatch that excites the antisymmetric (quadrupole) mode, and (iii) a mismatch that excites the symmetric envelope mode. These cases are shown in Figs. 1-3, respectively. We see that the phase-space halo parameters $H x$,Hy and the spatial-profile parameters $h_{x}, h_{y}$ are ap- proximately constant at values less than 1 for the matched beam. This is consistent with the lack of significant halo in the $x-x^{\prime}$ phase-space plot and the $x$ profile plot shown in Fig. 4. Similar results are obtained in $y-y^{\prime}$ and $y$ (not shown). When the beam is mismatched, the $H$ parameters smoothly increase while the $h$ parameters are oscillatory about an increasing mean. Both the $h$ and $H$ halo parameters assume values larger than 1, indicating strong halo formation; this result is consistent with the $x$ - $x^{\prime}$ phase-space plots and $x$-profile plots, shown for the symmetric mismatched case in Fig. 5.
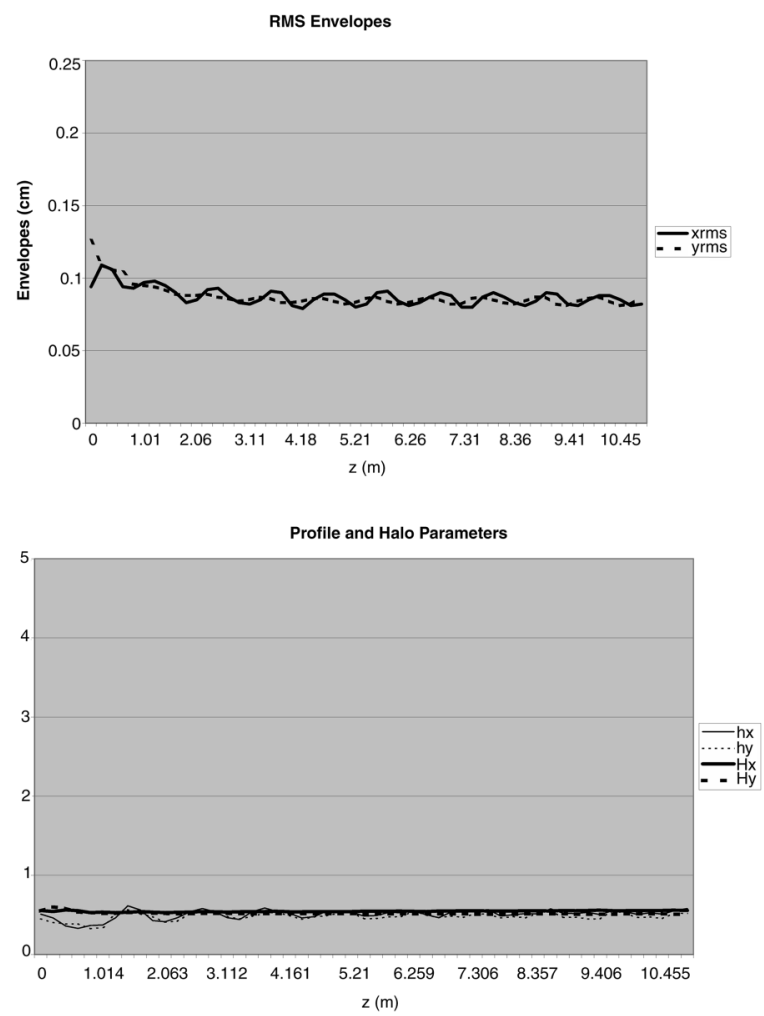

FIG. 1. rms envelopes and halo parameters for the matched case. 

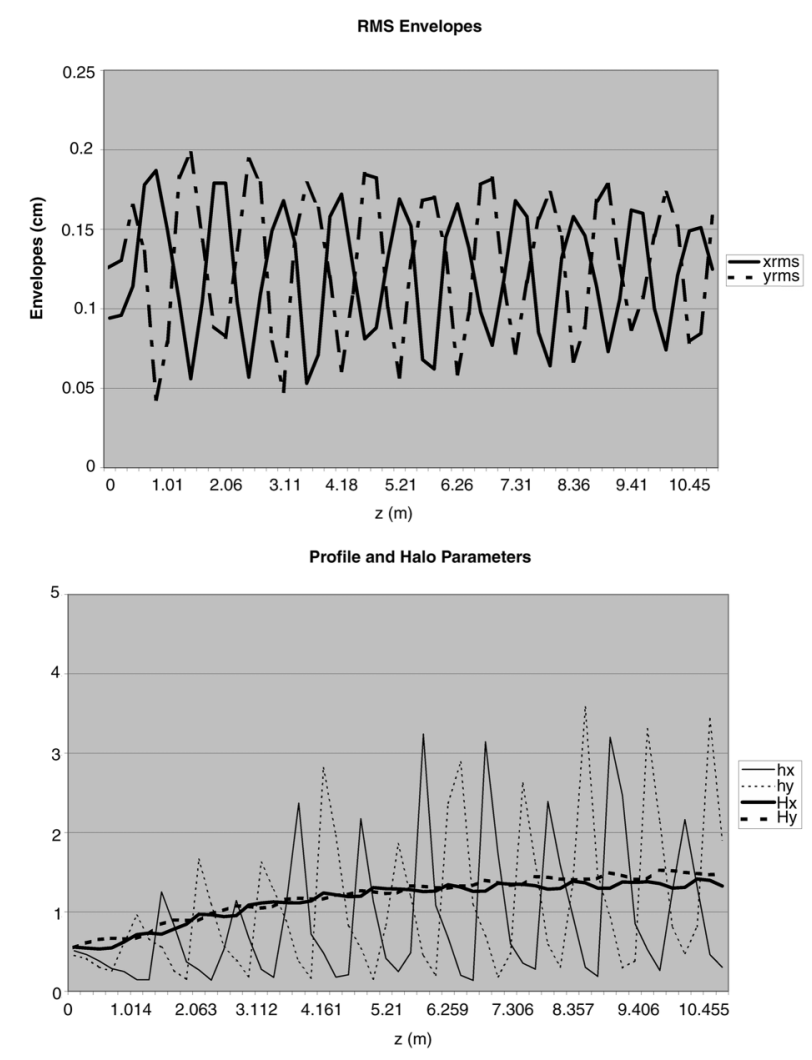

FIG. 2. rms envelopes and halo parameters for the quadrupole mismatch.
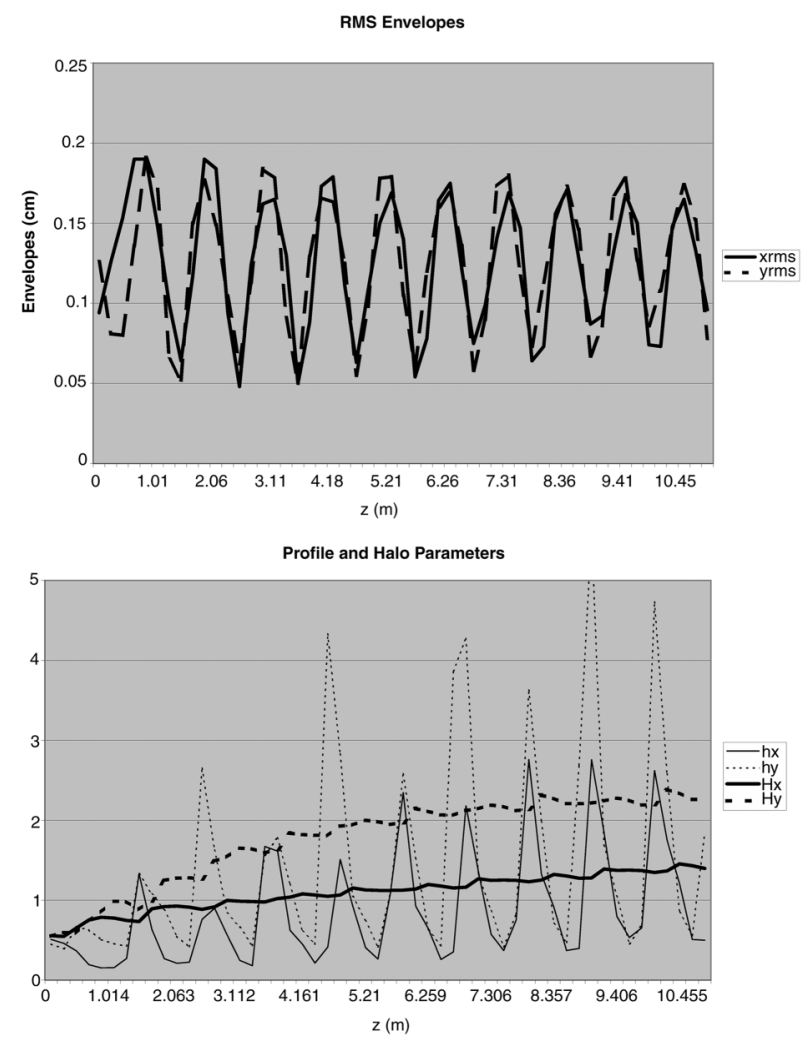

FIG. 3. rms envelopes and halo parameters for the symmetric mismatch.
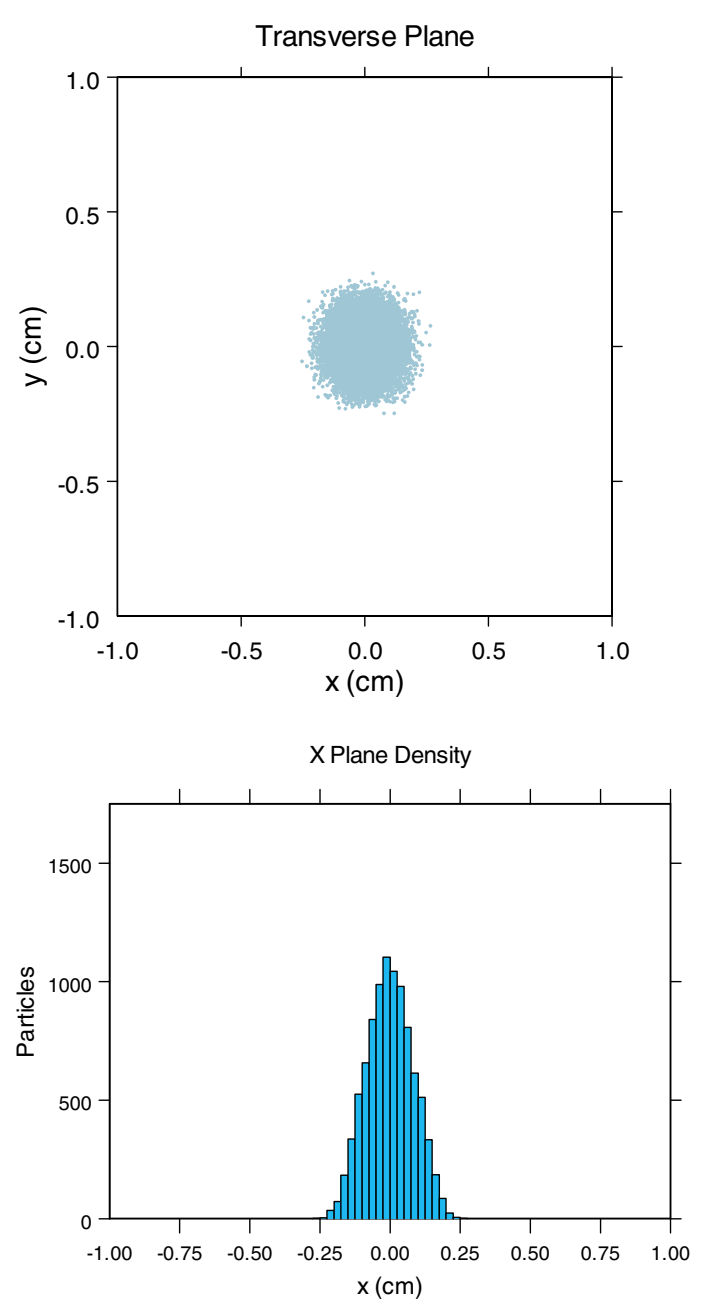

FIG. 4. (Color) Matched beam.

Unlike the idealized case of elliptical symmetry where $h=H$, the mismatched beams in Figs. 2 and 3 show that the parameters can be quite different for more general distributions seen in multiparticle simulation. As mentioned above, it has been observed that the halo can "hide," so that it is not observed in some spatial projections. The variations of the profile parameters $h$ are reflecting this fact, oscillating about the smoothly varying $H$ parameters.

\section{CONCLUSION}

The halo parameters defined here represent a convenient and model-independent method for quantifying the magnitude of beam halo observed in phase space and spatial projections. The spatial-profile parameter $h$ is, in essence, the kurtosis of the beam distribution normalized to the uniform distribution rather than the Gaussian. The phase-space halo parameter $H$ can be interpreted as a generalization of $h$ into 2D phase space such that it is invariant under linear forces. Both parameters are useful. 

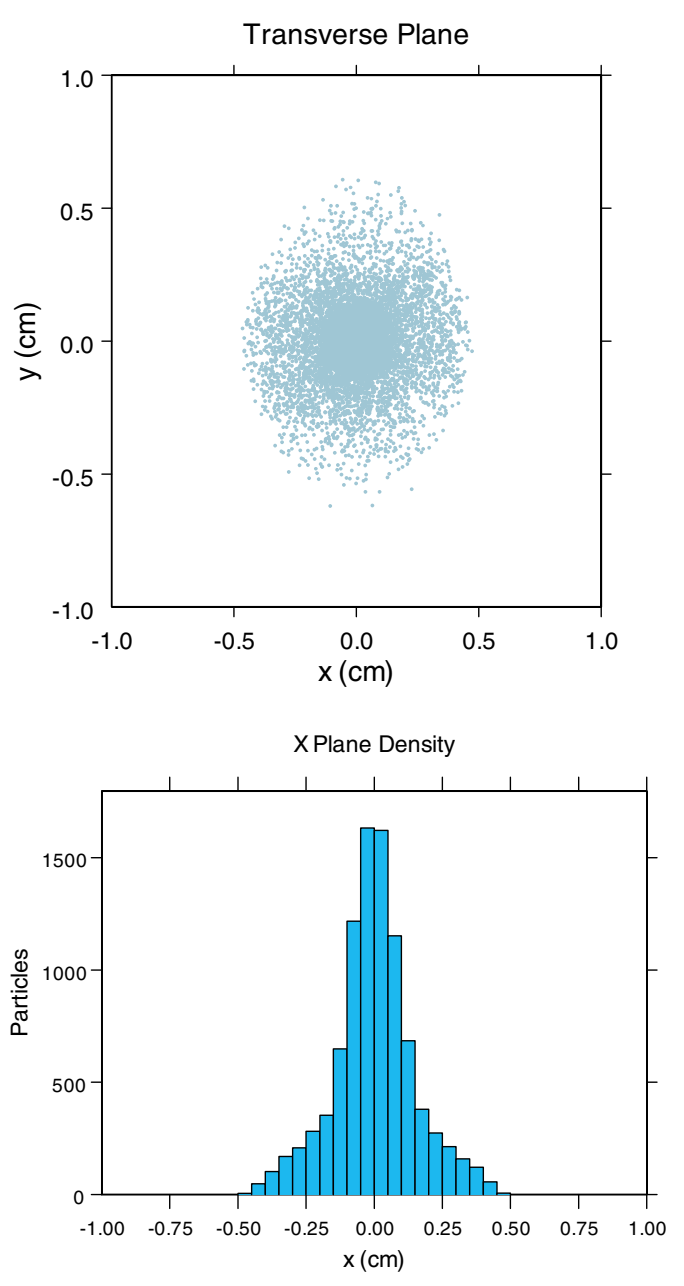

FIG. 5. (Color) Symmetric mismatch.

Although the profile parameter and halo parameter both reduce to the same value when the distribution has the elliptically symmetric form of Eq. (4), in general these parameters are not equal. As we saw in the simulation examples, halo observed in the 1D spatial profiles is relatively easily measured, but is oscillatory as the beam distribution evolves, and can hide as the distribution rotates in phase space. This behavior produces oscillations in the $h$ parameter. The 2D phase-space halo is more difficult to measure, but the $H$ parameter varies more smoothly as the halo evolves. It provides a more reliable characterization of the halo as an intrinsic property of the beam.

\section{ACKNOWLEDGMENTS}

We wish to thank Nicolas Pichoff for suggesting kinematic invariants in the halo parameter definition. This work was supported by the U.S. Department of Energy, LA-UR-02-4133.

\section{APPENDIX}

The moments of a distribution described by Eq. (4) may be computed using a technique similar to that of Dragt et al. [9]. Specifically, let

$$
\left\langle q^{m} p^{n}\right\rangle=\frac{1}{N} \int_{0}^{\infty} q^{m} p^{n} f\left[z^{T} Q z\right] d^{2} z,
$$

where

$$
\begin{gathered}
N=\int_{0}^{\infty} f\left[z^{T} Q z\right] d^{2} z, \quad Q \equiv\left(\begin{array}{cc}
\gamma & \alpha \\
\alpha & \beta
\end{array}\right), \quad z \equiv\left(\begin{array}{c}
q \\
p
\end{array}\right), \\
d^{2} z \equiv d q d p .
\end{gathered}
$$

The matrix $Q$ is symmetric and positive definite. Thus, there is an $R \in S O(2)$ which diagonalizes $Q$, say

$$
\Lambda=R^{T} Q R=\left(\begin{array}{cc}
\lambda_{1} & 0 \\
0 & \lambda_{2}
\end{array}\right),
$$

where $\lambda_{1}, \lambda_{2}$ are the eigenvalues of $Q$. Note that

$$
\operatorname{det} \Lambda=\lambda_{1} \lambda_{2}=\operatorname{det} Q=\beta \gamma-\alpha^{2}=1,
$$

where the last relation is a property of the Courant-Snyder parameters. With the substitution $\zeta=R^{T} z$ and the fact that $\operatorname{det} R=1$, we have

$$
\begin{aligned}
\left\langle q^{m} p^{n}\right\rangle= & \frac{1}{N} \int_{0}^{\infty}\left(R_{11} \zeta_{1}+R_{12} \zeta_{2}\right)^{m}\left(R_{21} \zeta_{1}+R_{22} \zeta_{2}\right)^{n} \\
& \times f\left[\zeta^{T} \Lambda \zeta\right] d^{2} \zeta .
\end{aligned}
$$

Using the additional substitution

$$
\begin{gathered}
\zeta_{1}=\frac{r}{\sqrt{\lambda_{1}}} \cos \theta, \quad \zeta_{2}=\frac{r}{\sqrt{\lambda_{2}}} \sin \theta, \\
d^{2} \zeta=\frac{r d r d \theta}{\sqrt{\lambda_{1} \lambda_{2}}}=r d r d \theta,
\end{gathered}
$$

we obtain the formula

$$
\begin{aligned}
\left\langle q^{m} p^{n}\right\rangle= & \frac{F_{\frac{m+n}{2}}}{F_{0}} \frac{1}{2 \pi} \int_{0}^{\infty}\left(\frac{R_{11}}{\sqrt{\lambda_{1}}} \cos \theta+\frac{R_{12}}{\sqrt{\lambda_{2}}} \sin \theta\right)^{m} \\
& \times\left(\frac{R_{21}}{\sqrt{\lambda_{1}}} \cos \theta+\frac{R_{22}}{\sqrt{\lambda_{2}}} \sin \theta\right)^{n} d \theta,
\end{aligned}
$$

where

$$
F_{k} \equiv \int_{0}^{\infty} s^{k} f(s) d s
$$

The above formula can be used to compute all the moments and, thus, the kinematic invariants, in terms of the $F_{k}$.

[1] R. Gluckstern, Phys. Rev. Lett. 73, 1247 (1994).

[2] R. L. Gluckstern, A.V. Fedotov, S. Kurennoy, and R. Ryne, Phys. Rev. E 58, 4977-4990 (1998).

[3] T. P. Wangler and K. R. Crandall, Phys. Rev. ST Accel. Beams 1, 084201 (1998).

[4] T. S. Wang, Phys. Rev. E 61, 855-861 (2000). 
[5] C. K. Allen, in Proceedings of the XX International Linac Conference, 2000, edited by A.W. Chao (SLAC Report No. SLAC-R-561, eConf: C000821, 2000).

[6] T. P. Wangler and K. R. Crandall, in Proceedings of the XX International Linac Conference, 2000 (Ref. [5]).

[7] W. P. Lysenko and M. Overley, in Linear Accelerators and Beam Optics Codes, edited by C. R. Eminhizer, AIP Conf. Proc. No. 177 (AIP, New York, 1988), pp. 323-335.
[8] D. D. Holm, W. P. Lysenko, and J. C. Scovel, J. Math. Phys. (N.Y.) 31, 1610-1615 (1990).

[9] A. J. Dragt, R. L. Gluckstern, F. Neri, and G. Rangarajan, in Frontiers of Particle Beams; Observations, Diagnosis and Corrections, edited by M. Month and S. Turner, Lecture Notes in Physics Vol. 343 (Springer-Verlag, Berlin, 1988), pp. 94-121.

[10] C. K. Allen and T.P. Wangler, in Proceedings of the PAC2001, Chicago, edited by P. Lucas and S. Webber (IEEE Cat. No. 01CH37268, 2001), p. 1732. 